$211 \mid 2013$

Varia

\title{
Juhé-Beaulaton, Dominique (dir.) - Forêts sacrées et sanctuaires boisés
}

Julien Bondaz

\section{(2) OpenEdition}

1 Journals

Édition électronique

URL : http://journals.openedition.org/etudesafricaines/14519

DOI : 10.4000/etudesafricaines. 14519

ISSN : 1777-5353

Éditeur

Éditions de l'EHESS

\section{Édition imprimée}

Date de publication : 16 septembre 2013

Pagination : 752-754

ISBN : 978-2-7132-2388-4

ISSN : 0008-0055

Référence électronique

Julien Bondaz, "Juhé-Beaulaton, Dominique (dir.) - Forêts sacrées et sanctuaires boisés », Cahiers d'études africaines [En ligne], 211 | 2013, mis en ligne le 20 septembre 2013, consulté le 23 septembre 2020. URL : http://journals.openedition.org/etudesafricaines/14519; DOI : https://doi.org/10.4000/ etudesafricaines. 14519

Ce document a été généré automatiquement le 23 septembre 2020.

(c) Cahiers d'Études africaines 


\title{
Juhé-Beaulaton, Dominique (dir.) - Forêts sacrées et sanctuaires boisés
}

\author{
Julien Bondaz
}

\section{RÉFÉRENCE}

JUHÉ-BEAULATON, Dominique (dir.) - Forêts sacrées et sanctuaires boisés. Des créations culturelles et biologiques (Burkina Faso, Togo, Bénin). Paris, Karthala, 2010, 280 p., bibl.

1 L'ouvrage collectif dirigé par Dominique Juhé-Beaulaton s'inscrit à la fois dans le cadre des recherches sur les bois sacrés qui connaissent (comme l'introduction le montre bien) un véritable essor à partir du début des années 1990, autour du texte fondateur et plusieurs fois discuté ici de Michel $\mathrm{Cartry}^{1}$, et dans celui des réflexions récentes concernant les patrimonialisations de la nature en Afrique ${ }^{2}$. l'étude des «forêts sacrées et sanctuaires boisés » révèle ainsi un objet problématique, un concept dont la vertu descriptive doit être interrogée. Le choix d'un comparatisme à petite échelle (les études de cas concernent le Burkina Faso, le Togo et le Bénin) se révèle à ce sujet particulièrement heuristique. En proposant de parler de «bois sacré », de "forêt sacrée ", de "sanctuaire boisé », mais aussi de "lieu excepté » ou de «fragments forestiers ", les auteurs rassemblés ici montrent comment le mode de désignation et les enjeux de traduction des termes vernaculaires soulèvent des questions théoriques.

2 L'idée d'en finir avec l'illusion d'une « virginité floristique » (p. 72) associée à l'idée de "bois sacré " ${ }^{3}$ constitue cependant un objectif commun et invite à un "exercice des regards croisés»(p.73), en rassemblant des chercheurs de disciplines différentes: anthropologues, historiens, écologues ou botanistes. Un tel choix conduit à la production de données variées sur les «bois sacrés ", depuis les listes et les statistiques des espèces ligneuses qui les composent jusqu'aux gestes rituels qui participent à leur agencement ou à leur gestion. Les typologies proposées sont ainsi susceptibles d'être organisées aussi bien sur la base des types écofloristiques, comme le proposent notamment Kouami Kokou et Adzo Dzifa Kokutse, que sur celle des usages, des 
prescriptions ou des interdits dont les bois sacrés font l'objet, ou sur la distinction entre «bonnes » et «mauvaises » forêts. Il s'agit là, pour reprendre le sous-titre du texte de Stéphan Dugast, d'« un domaine d'exercice de la pensée classificatoire », et il est à ce sujet notable que les classifications écologiques et symboliques des bois sacrés ne se recoupent pas nécessairement. Plus exactement, comme le note S. Dugast, la question de «savoir si les modes de classification associés ont leur projection dans le domaine de la composition floristique des sites concernés » reste largement ouverte (p. 182), et c'est l'une des leçons de l'ouvrage.

3 Mais l'intérêt majeur des études rassemblées réside dans la richesse et la finesse des données collectées, notamment celles fournies par les enquêtes ethnographiques. Il s'agit à chaque fois de prendre au sérieux les savoirs locaux concernant les bois sacrés. Les différents cas étudiés apportent plusieurs enseignements. En premier lieu, le rôle joué par les bois sacrés dans la production des territoires, selon des modalités variées, possède évidemment une place importante dans les analyses proposées, et permet de mettre en évidence la fonction liminale de certains d'entre eux (Chiara Alfieri parle même de «sas » à leur sujet). Les "processus de territorialisation d'une communauté villageoise» (p.60) sont mis en évidence, et la question de l'autochtonie apparait souvent centrale. Le texte que signe Danouta Liberski-Bagnoud avec Anne Fournier et Saïbou Nignan insiste particulièrement sur cet aspect. Les idées développées par la première dans son livre, Les Dieux du territoire. Penser autrement la généalogie ${ }^{4}$, invitent ici à une réflexion pluridisciplinaire. Au-delà, dans la plupart des contributions, les relations entre les bois sacrés et les récits de fondation ou d'origine, entre ancrage territorial et histoire locale ou des migrations, entre topologie et généalogie, sont bien développées.

En effet, la mise en réseau des différents bois sacrés, parfois actualisée par la circulation de certains rituels ou par leur enchâssement, voire par des pratiques rituelles de bouturage, rend compte des classes d'entités invisibles qui les habitent, des découpages territoriaux et des relations entre familles ou lignages, sur un mode souvent hiérarchique. l'enjeu est de comprendre non seulement les relations entre bois sacrés, entités invisibles, portions de territoire et lignages mais aussi les relations des bois sacrés (et donc des entités invisibles, des territoires, des lignages) entre eux. Pour le dire autrement, il s'agit de mettre en évidence le « complexe relationnel» (p. 268) dans lequel ils s'insèrent, et ce à différentes échelles, depuis celle du quartier villageois (l étude de Mathilde Lainé est particulièrement révélatrice à ce sujet) à celle des entités territoriales les plus grandes: par exemple, comme le note D. Juhé-Beaulaton, "l'histoire des relations entre les royaumes et les chefferies de l'aire Aja-Fon s'est souvent traduite par la création de nouveaux sites sacrés » (p. 28). Les cas de bois sacrés fonctionnant comme des marqueurs de frontière ou comme des "lieux liminaux" (p. 153) sont également tout à fait intéressants. Klaus Hamberger en particulier montre comment, en pays ouatchi, au Togo, les termes de la parenté agnatique et ceux de la parenté utérine permettent de penser la constitution d'un groupe local et la relation à l'étranger, à travers les rapports que les hommes entretiennent avec les «vodous trouvés » et leurs "forêts ». l'approche structuraliste se révèle ici pertinente ${ }^{5}$. Plus largement, les déplacements, transferts, abandons ou réemplois de bois sacrés, en fonction du contexte, notamment politique (lutte contre les pratiques animistes au Bénin, migrations des populations, nouveaux enjeux liés aux patrimonialisations ou à la mise en tourisme), sont révélateurs de dynamiques historiques et sociales complexes. 
Le cas de la forêt sacrée de Kpassé au Bénin, analysé par D. Juhé-Beaulaton, paraît à ce titre exemplaire.

Le fait que les bois sacrés fonctionnent à la fois comme des lieux de pouvoir et comme des lieux de mémoire conduit en outre les auteurs à aborder souvent finement les problèmes liés à leur patrimonialisation. Il est d'ailleurs heureux qu ils ne soient pas analysés trop mécaniquement comme des points de jonction entre la question de la biodiversité et celle de la diversité culturelle. De ce point de vue, une distance est clairement prise avec les discours souvent tenus par les institutions patrimoniales internationales ou les instances officielles des pays concernés au sujet du patrimoine naturel. Les auteurs mettent en garde contre une définition ethnocentriste du patrimoine ou de la biodiversité (p. 61) et insistent au contraire sur les tensions entre la logique coloniale de mise en réserve de domaines forestiers et les pratiques locales de sanctuarisation se traduisant par des usages rituels, par exemple au Bénin ou au Togo (p. 35), ou plus largement entre une logique patrimoniale naturaliste et celle du réseau des analogies qui caractérise nombre de représentations locales concernant les bois sacrés. Les différents contributeurs montrent bien comment des chaînes analogiques s'organisent, selon des modalités parfois différentes et s'inscrivant souvent selon un principe métonymique, entre territoires, bois sacrés, entités invisibles et responsables de culte. Le texte de Marie Daugey est sans doute celui qui rend le mieux compte de ces rapports analogiques, invalidant l'idée selon laquelle les bois sacrés seraient des "morceaux de nature ". Nous sommes ici en effet au cœur du problème, puisque la qualité des études de cas présentés dans cet ouvrage, agrémenté de nombreuses illustrations, permet clairement de dépasser l'analyse des particularismes et la construction d'un objet d'étude complexe pour proposer un comparatisme heuristique, échappant au double écueil d'un symbolisme diffus ou sans histoire et d'une naturalisation trop forte du patrimoine dit naturel.

\section{NOTES}

1. M. CARTRY, «Les bois sacrés des autres : les faits africains ", in Les Bois sacrés, Actes du colloque international de Naples, Collection du centre Jean Bérard, 10, 1993, pp. 193-208.

2. Voir en particulier les deux ouvrages dirigés par M.-C. CORMIER-SALEM, D. JUHÉ-BEAULATON, J. BoutraIS \& B. RousSEL, Patrimonialiser la nature tropicale. Dynamiques locales, enjeux internationaux, Paris, IRD, 2002, et Patrimoines naturels au Sud. Territoires, identités et stratégies locales, Paris, IRD, 2005, auxquels plusieurs auteurs ici rassemblés ont contribué.

3. Pour une plus grande commodité de lecture, cette expression seule sera utilisée dans ce compte rendu.

4. D. LIBERSKI-BAGNOUD, Les Dieux du territoire. Penser autrement la généalogie, Paris, Éditions du CNRSÉditions de la Maison des sciences de l'homme, 2002.

5. Cette approche est développée par l'auteur dans un ouvrage paru plus récemment: K. HAMBERGER, La parenté vodou. Organisation sociale et logique symbolique en pays ouatchi (Togo), Paris, Éditions du CNRS-Éditions de la Maison des sciences de l'homme, 2011. 